$06 ; 13$

\title{
Исследование многослойных тонкопленочных структур методом резерфордовского обратного рассеяния
}

\author{
() В.И. Бачурин, Н.С. Мелесов, Е.О. Паршин, А.С. Рудый, А.Б. Чурилов \\ Ярославский филиал Физико-технологического института им. К.А. Валиева РАН, \\ Ярославль, Россия \\ E-mail: vibachurin@mail.ru
}

Поступило в Редакцию 20 марта 2019 г.

В окончательной редакции 20 марта 2019 г.

Принято к публикации 25 марта 2019 г.

Представлены результаты изучения возможностей метода резерфордовского обратного рассеяния для анализа многослойной структуры, содержащей слои нанометрового масштаба с близкими по массам элементами. Показано, что резерфордовское обратное рассеяние позволяет с достаточно высокой точностью определять состав таких структур, толщину пленки в целом и толщины отдельных слоев и может использоваться для входного контроля технологических структур, применяемых в микро- и нанотехнологиях.

Ключевые слова: многослойные тонкопленочные структуры, послойный анализ, резерфордовское обратное рассеяние.

DOI: 10.21883/PJTF.2019.12.47914.17798

Многослойные тонкопленочные структуры находят широкое применение в микро- и нанотехнологиях. Для оптимизации свойств таких пленок, зависящих от химического состава слоев и их толщин, необходимо иметь информацию о распределении элементов по глубине, а также о толщине и плотности слоев пленки. Эти данные можно получать, используя как разрушающие поверхность образца методы (вторично-ионная массспектрометрия, растровая электронная оже-спектроскопия и др.), так и неразрушающие методы анализа (резерфордовское обратное рассеяние, РОР) [1,2]. К преимуществам первых относится достаточно хорошее разрешение по глубине, которое при подборе условий ионной бомбардировки может составлять единицы нанометров. Однако ионное перемешивание и развитие топографического рельефа при распылении приводят к ухудшению послойного разрешения при увеличении числа слоев, а проведение количественного анализа требует применения эталонных образцов. РОР является неразрушающим количественным методом анализа поверхности и широко применяется при изучении химического состава и толщин пленок субмикронного масштаба. К недостаткам метода можно отнести невысокое послойное разрешение $(5-15 \mathrm{~nm})$ и трудности, возникающие при анализе структур с близкими по массе элементами. Но имеющиеся возможности моделирования экспериментальных спектров позволяют получать надежную информацию при послойном анализе многослойных тонкопленочных структур нанометрового масштаба, содержащих элементы с близкими массами $[3,4]$.

В работе представлены результаты изучения возможностей метода РОР для анализа таких структур. В качестве образца использовались многослойные пленки, полученные методом магнетронного напыления. В табл. 1 представлены толщины слоев и содержание в них элементов. Числа перед составом слоя указывают его толщину в нанометрах по данным технологического процесса. Подобного типа пленки используются в настоящее время для создания магнитно-туннельных переходов с высокими функциональными характеристиками. Встраивание их в последующие схемы требует проведения более 20 технологических операций. Поэтому желательно проведение входного контроля подобных структур на предмет состава и толщины слоев. Анализ, проведенный ранее с помощью вторично-ионной масс-спектрометрии, не позволил получить достоверную информацию о распределении элементов в слоях и их толщинах в пленке [5].

Исследование образцов проводилось на установке K2MV (HVEE). Использовались ионы $\mathrm{He}^{+}$с энергией 1.2 и $1.9 \mathrm{MeV}$ и ионы $\mathrm{He}^{++}$с энергией $3.1 \mathrm{MeV}$. Угол рассеяния ионов во всех экспериментах составлял $\theta=165^{\circ}$, угол падения ионного пучка на образец при энергии $1.2 \mathrm{MeV}$ был равен $\alpha=30^{\circ}$ (угол выхода из образца $\left.\beta=45^{\circ}\right)$, при остальных энергиях $\alpha=8.6^{\circ}\left(\beta=22.5^{\circ}\right)$. Для создания модели образца в точке $P 1$ (рис. 1) регистрировались спектры обратнорассеянных ионов при различных экспериментальных условиях. Из-за различных параметров измерений на этих спектрах происходит наложение сигналов элементов в отличающихся комбинациях, что использовалось для подбора модели, удовлетворяющей всем спектрам сразу [6]. Моделирование экспериментальных спектров проводилось с помощью программы SIMNRA [7].

При рассеянии ионов $\mathrm{He}^{++}$с энергией $3094 \mathrm{keV}$ удалось практически полностью разрешить сигналы от $\mathrm{Ru}$ (слои 1 и 6) и от внутренних слоев Та (слои 10 и 12). В спектрах, полученных при рассеянии ионов $\mathrm{He}^{+}$с энергией 1237 и $1856 \mathrm{keV}$, большинство пиков соседних элементов накладывается друг на друга, но из них 
Таблица 1. Структура и состав образца по данным технологического процесса напыления пленки и результатам моделирования спектров РОР

\begin{tabular}{|c|c|c|c|c|c|}
\hline \multirow{3}{*}{ Номер слоя } & \multirow{3}{*}{ Слой } & \multicolumn{3}{|c|}{ Состав } & \multirow{3}{*}{ Толщина, $10^{15}$ atom $/ \mathrm{cm}^{2}(\mathrm{~nm})$} \\
\hline & & \multirow{2}{*}{ Элемент } & \multicolumn{2}{|c|}{ Содержание } & \\
\hline & & & at. $\%$ & $10^{15}$ atom $/ \mathrm{cm}^{2}$ & \\
\hline 1 & 2 & 3 & 4 & 5 & 6 \\
\hline 1 & $7 \mathrm{Ru}$ & $\mathrm{Ru}$ & 100 & 51.479 & $51.479(6.96)$ \\
\hline 2 & $10 \mathrm{Ta}$ & $\mathrm{Ta}$ & 100 & 51.512 & $51.512(9.30)$ \\
\hline 3 & $2.5 \mathrm{Co}_{0.60} \mathrm{Fe}_{0.20} \mathrm{~B}_{0.20}$ & $\begin{array}{c}\mathrm{Co} \\
\mathrm{Fe} \\
\mathrm{B}\end{array}$ & $\begin{array}{l}60 \\
20 \\
20\end{array}$ & $\begin{array}{r}10.5 \\
3.5 \\
3.5\end{array}$ & 17.5 \\
\hline 4 & $2 \mathrm{MgO}$ & $\begin{array}{c}\mathrm{Mg} \\
\mathrm{O}\end{array}$ & $\begin{array}{l}50 \\
50\end{array}$ & $\begin{array}{l}10.1 \\
10.1\end{array}$ & $20.2 \quad(1.89)$ \\
\hline 5 & $2.5 \mathrm{Co}_{0.60} \mathrm{Fe}_{0.20} \mathrm{~B}_{0.20}$ & $\begin{array}{c}\mathrm{Co} \\
\mathrm{Fe} \\
\mathrm{B}\end{array}$ & $\begin{array}{l}60 \\
20 \\
20\end{array}$ & $\begin{array}{r}10.5 \\
3.5 \\
3.5\end{array}$ & 17.5 \\
\hline 6 & $0.9 \mathrm{Ru}$ & $\mathrm{Ru}$ & 100 & 5.904 & $5.904(0.8)$ \\
\hline 7 & $2.0 \mathrm{Co}_{0.70} \mathrm{Fe}_{0.30}$ & $\begin{array}{l}\mathrm{Co} \\
\mathrm{Fe}\end{array}$ & $\begin{array}{l}63.1557 \\
36.8443\end{array}$ & $\begin{array}{r}15.343 \\
8.951\end{array}$ & 24.294 \\
\hline 8 & $16 \mathrm{Ir}_{0.18} \mathrm{Mn}_{0.82}$ & $\begin{array}{c}\mathrm{Ir} \\
\mathrm{Mn}\end{array}$ & $\begin{array}{l}24.3649 \\
75.6351\end{array}$ & $\begin{array}{l}26.632 \\
82.673\end{array}$ & 109.305 \\
\hline 9 & $3 \mathrm{NiFe}$ & $\begin{array}{l}\mathrm{Ni} \\
\mathrm{Fe}\end{array}$ & $\begin{array}{l}62.6456 \\
37.3544\end{array}$ & $\begin{array}{l}20.435 \\
12.185\end{array}$ & 32.620 \\
\hline 10 & $5 \mathrm{Ta}$ & $\mathrm{Ta}$ & 100 & 26.555 & $26.555(4.79)$ \\
\hline 11 & $30 \mathrm{CuN}$ & $\begin{array}{l}\mathrm{Cu} \\
\mathrm{N}\end{array}$ & $\begin{array}{l}87.1679 \\
12.8321\end{array}$ & $\begin{array}{c}230.471 \\
33.928\end{array}$ & 264.399 \\
\hline 12 & $5 \mathrm{Ta}$ & $\mathrm{Ta}$ & 100 & 26.552 & $26.552(4.79)$ \\
\hline
\end{tabular}

удалось выделить уровень сигнала Со и положение экстремума пика Ir.

Получение информации о легких элементах в образце $(\mathrm{B}, \mathrm{N}, \mathrm{O}, \mathrm{Mg})$ затруднено, поскольку их сигналы сравнимы с уровнем шума. Однако использование ионов $\mathrm{He}^{++}$ с энергией $3052 \mathrm{keV}$ позволило оценить содержание О в слое $\mathrm{MgO}$ с помощью резонансного ядерного рассеяния, сечение которого более чем в 20 раз превышает резерфордовское сечение рассеяния [8].

В табл. 1 представлены состав и толщины слоев пленки. В столбце 1 указан номер слоя от поверхности, в столбце 2 - толщина и химический состав слоя по данным технологического процесса осаждения пленки, в столбце 3 - элементы, содержащиеся в слое, в столбцах 4 и 5 - содержание элементов в слое, а в столбце 6 - толщина слоев по результатам моделирования. При переводе толщины слоев из принятой в методе POP шкалы atom $/ \mathrm{cm}^{2}$ в $\mathrm{nm}$ плотность моноэлементных слоев и слоя оксида магния принималась равной плотности массивных материалов. Количество $\mathrm{Ru}$ и Та в слоях определено с ошибкой, не превышающей $\pm 5 \%$, а $\mathrm{O}$ - с точностью не хуже $\pm 9 \%$. Оценка погрешности в определении содержания остальных элементов в образце затруднительна, поскольку при построении модели изменение содержания одного элемента может компенсироваться изменением содержания другого.

Из табл. 1 видно, что химический состав слоев модельного образца заметно отличается от заявленного начиная со слоя 7. Отметим низкое содержание $\mathrm{N}$ в слое $\mathrm{CuN}$. По данным рентгеноструктурного анализа, выполненного в [5], в слое 11 присутствует стехиометрический $\mathrm{Cu}_{3} \mathrm{~N}$. По результатам РОР-анализа можно заключить, что состав этого слоя представляет собой смесь чистой меди и нитрида меди $\left(\mathrm{Cu}_{3} \mathrm{~N}\right)$. Различия состава в многокомпонентных слоях могут быть связаны с подгонкой модели к экспериментальным спектрам. Видно, что толщины слоев и их состав, измеренные по результатам анализа спектров РОР, довольно близки в большинстве случаев к данным технологического процесса напыления структуры.

Для исследования возможности контроля изменения толщины по образцу проводились сравнения спектров, полученных в различных точках пленки, расположенных вблизи края пластины (рис. 1). На рис. 2 представлены низкоэнергетические и высокоэнергетические части спектров РОР, полученные в точках $P 1, P 3$, 


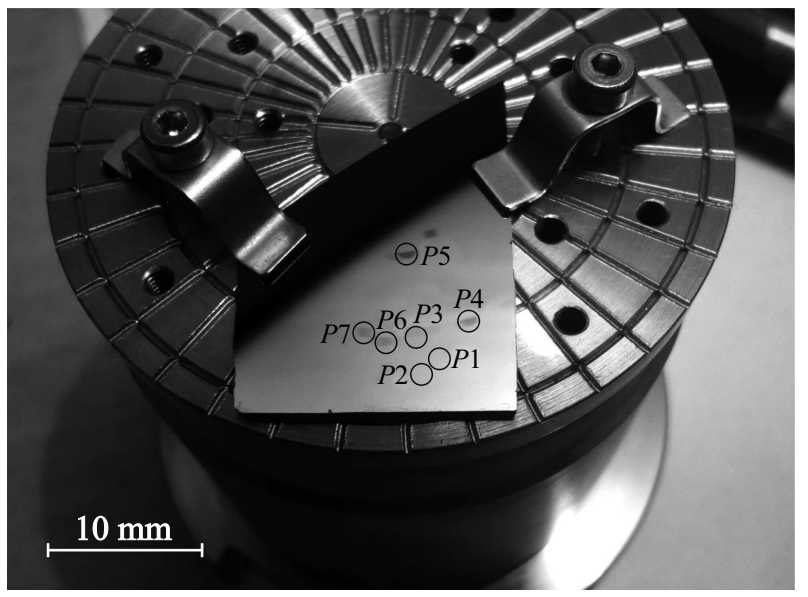

Рис. 1. Расположение точек многослойной структуры вблизи края пластины $\mathrm{SiO}_{2} / \mathrm{Si}$, в которых регистрировались спектры POP.
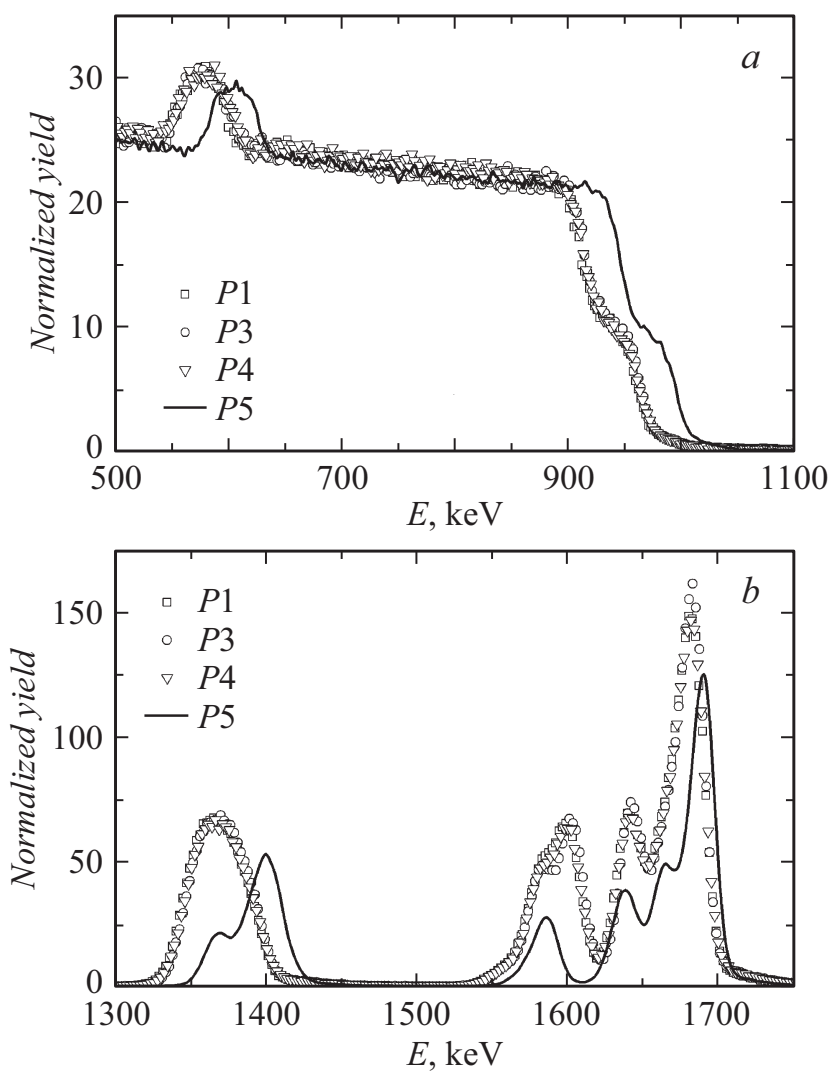

Рис. 2. Сравнение низкоэнергетической $(a)$ и высокоэнергетической $(b)$ частей спектров РОР, полученных в точках $P 1$, $P 3, P 4$ и $P 5$, при энергии зондирующих ионов $\mathrm{He}^{+}$, равной $1856 \mathrm{keV}$.

$P 4, P 5$. Здесь и далее значения по оси ординат приводятся в нормированных единицах: выходной сигнал многоканального анализатора (МКА) делился на дозу экспозиции (в $\mu \mathrm{C})$, телесный угол детектора (в sr) и ширину канала MКА (в $\mathrm{eV})$. Точка $P 3$ расположена
Таблица 2. Сравнение толщины пленки в различных точках образца

\begin{tabular}{c|c|c|c|c}
\hline $\begin{array}{c}\text { № } \\
\text { п/п }\end{array}$ & Точка & $\begin{array}{c}\text { Расстояние } \\
\text { до края, } \mathrm{mm}\end{array}$ & Энергия, $\mathrm{keV}$ & Толщина, \% \\
\hline 1 & $P 1$ & 9.0 & $1237,3094,1856$ & 100 \\
2 & $P 2$ & 9.0 & 3054 & 100 \\
3 & $P 4$ & 8.4 & 1856 & $99.7 \pm 0.3$ \\
4 & $P 3$ & 7.3 & 1856 & $98.9 \pm 0.4$ \\
5 & $P 6$ & 6.4 & 3094 & $95.7 \pm 0.4$ \\
6 & $P 7$ & 4.8 & 1237 & $91.4 \pm 0.7$ \\
7 & $P 5$ & 2.5 & 1856 & $65.5 \pm 0.8$
\end{tabular}

недалеко от точки $P 1$, но несколько ближе к краю напыления. Точка $P 4$ расположена на большом удалении от точки $P 1$, но на примерно равном с ней расстоянии от края напыления. Точка P5 была взята вблизи края напыления.

Детальный анализ спектров, полученных при различных экспериментальных условиях, показывает, что спектры РОР в точках $P 4$ и $P 1$ практически совпадают. Для спектра, полученного в точке $P 3$, наблюдается смещение сигнала от глубоких слоев относительно спектра в точке $P 1$. Особенно отчетливо это заметно по смещению в область более высоких энергий сигнала Та слоя 12. Данный факт свидетельствует об интегральном уменьшении толщины пленки, наблюдение дифференциального уменьшения толщины каждого слоя выходит за пределы точности измерения. На спектpe, полученном в точке $P 5$, отчетливо наблюдается уменьшение толщины как всей пленки, так и каждого слоя по отдельности, причем уменьшение толщины в данном случае существенно превосходит наблюдаемые изменения в точке $P 3$.

Если считать, что уменьшение толщины пленки при приближении к краю напыления происходит пропорционально во всех слоях модельной мишени, полученной для спектров, то можно получить количественные значения изменения толщины в каждой точке. При таком допущении толщина в точке $P 3$ меньше толщины в точке $P 1$ на $1.1 \pm 0.4 \%$, в точке $P 4-$ на $0.3 \pm 0.3 \%$, в точке $P 5-$ на $34.5 \pm 0.8 \%$.

На рис. 3 в качестве примера приведено сравнение экспериментального спектра (точка P5) со спектром, полученным на основе модели образца с уменьшенной на $34.5 \%$ толщиной. Наблюдается хорошее согласие экспериментального спектра со спектром модельного образца, что позволяет достаточно точно определять толщину тонкопленочной структуры. Более того, видно, что положение экстремума экспериментального сигнала последнего слоя тантала совпадает с модельным пиком, но высота его несколько ниже. Это может быть связано с некорректностью предположения пропорционального уменьшения толщины всех слоев при приближении к краю напыления. Наилучшего совпадения по высоте сигнала от последнего слоя удается добиться при умень- 


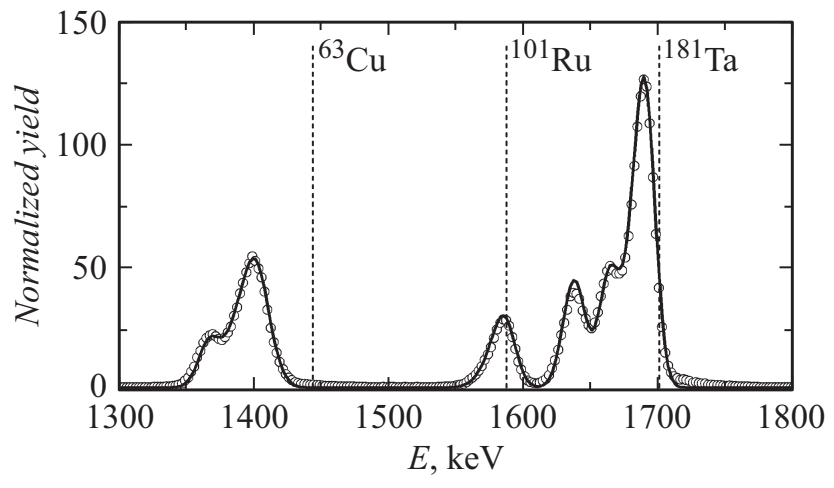

Pис. 3. Сравнение экспериментального спектра РОР в точке $P 5$ с симулированным спектром (линия), полученным на основе модели образца с уменьшенной на $34.5 \%$ толщиной.

шении его толщины на 43\%, а остальных по-прежнему на $34.5 \%$. В этом случае наилучшим оказывается и смещение сигнала от подложки. Этот факт может свидетельствовать о возможности оценивать не только толщину многослойной структуры, но и толщины отдельных слоев.

Данные по анализу толщины образца сведены в табл. 2. Точки, с которых регистрировались спектры, приводятся в порядке уменьшения расстояния до края напыления.

Таким образом, в результате проведенных экспериментальных исследований показано, что метод резерфордовского обратного рассеяния позволяет с достаточно высокой точностью определять состав многокомпонентных многослойных тонкопленочных структур нанометрового масштаба, толщину пленки в целом и толщины отдельных слоев и может использоваться для входного контроля технологических структур, применяемых в микро- и нанотехнологиях.

\section{Финансирование работы}

Работа выполнена в рамках государственного задания Министерства образования и науки РФ Ярославскому филиалу Физико-технологического института им. К.А. Валиева РАН по теме № 0066-2019-0003 на оборудовании Центра коллективного пользования „Диагностика микро- и наноструктур“.

\section{Конфликт интересов}

Авторы заявляют, что у них нет конфликта интересов.

\section{Список литературы}

[1] Oswald S., Baunack S. // Thin Solid Films. 2003. V. 425. P. 9-19. DOI: 10.1016/S0040-6090(02)01097-0

[2] Escobar Galindo R., Gago R., Lousa A., Albella J.M. // Trends Anal. Chem. 2009. V. 28. P. 494-505.

DOI: 10.1016/j.trac.2009.01.004
[3] Коломиеи В.Н., Кононенко И.Н., Кравченко С.Н., Захарец, М.И., Сторижко В.Е., Ввозный В.И., Бугай А.Н., Девизенко А.Ю. // Металлофизика и новейшие технологии. 2016. T. 38. № 6. С. 815-823. DOI: $10.15407 / \mathrm{mfint} .38 .06 .0815$

[4] Бачурин В.И., Мелесов Н.Н., Мироненко А.А., Паршин Е.О., Рудый А.С., Симакин С.Г., Чурилов А.Б. // Поверхность. 2019. № 4. С. 38-43. DOI: $10.1134 / \mathrm{S} 0207352819040024$

[5] Трушин О.С., Симакин С.Г., Васильев С.В., Смирнов Е.А. // Микроэлектроника. 2018. Т. 47. № 6. С. 424-430. DOI: $10.31857 / \mathrm{S} 054412690002768-1$

[6] Reis M.A., Chaves P.C., Corregitor V., Barradas N.P., Alves E., Dimroth F., Bett A.W. // X-ray Spectrom. 2005. V. 34. P. 372-375. DOI: $10.1002 /$ xrs. 841

[7] Mayer M. SIMNRA User's Guide. Garching, Germany: Max-Planck-Institut für Plasmaphysik, 2011. $220 \mathrm{p}$.

[8] Knapp J.A., Barbour J.C., Doyle B.L. // J. Vac. Sci. Technol. A. 1992. V. 10. P. 2685-2690. DOI: $10.1116 / 1.577959$ 